Title: Treating COVID-19 with Anticoagulation Based on Kabir Bleeding Risk Score

Author: Azad Kabir, MD MSPH (Biostatistics)

Running Title: Treating COVID-19 based on Kabir bleeding risk score

Corresponding author's name and contact information (e-mail address, mailing address, phone number): Azad Kabir, MD, MSPH, ABIM; Department of Research and Innovation; Doctor Ai, LLC; 1120 Beach Blvd, Biloxi; MS 39530; Department of Internal Medicine, Jackson Hospital, Montgomery, Alabama. Email: azad.kabir@gmail.com; Cell: 228-342-6278

Word count: 1392

Keywords: COVID-19, anticoagulation, bleeding risk, mechanism of death; treatment strategy, non-critically ill

Key points:

The author developed the Kabir bleeding risk score-based treatment strategies for COVID-19 which can be visited by clicking on the following link: <https://patient.ddxrx.com/anticoagulation.php>. 


\section{Abstract (165):}

Three large randomized clinical trials named the ATTACC, ACTIV-4a, and REMAP-CAP were terminated early as these trials showed use of therapeutic anticoagulation among non-critical COVID-19 patients increased the probability of survival to hospital discharge as well as reduced the need for cardiovascular or respiratory organ support. These clinical trials also showed when a COVID-19 patient presents with a critical stage, therapeutic anticoagulation does not provide any benefit. This study retrospectively evaluated the COVID-19 admission at Jackson Hospital, Alabama, USA from June 15th, 2020, to June 15th, 2021. The study developed COVID-19 mechanism of death and found that anticoagulation doses can be titrated up or down based on D-Dimer trends and many patients do not need therapeutic anticoagulation, rather an intermediate dose (Lovenox $0.5 \mathrm{mg} / \mathrm{kg}$ subQ BID or higher dose) anticoagulation can be sufficient for those who have a higher risk of bleeding. The author developed the Kabir bleeding risk scorebased treatment strategies for COVID-19 patients which can be visited by clicking on the following link: <https://patient.ddxrx.com/anticoagulation.php $>$.

\section{Introduction:}

Three largest randomized clinical trials named the ATTACC, ACTIV-4a, and REMAP-CAP were terminated early, and investigators reported on August 4th, 2021, that in noncritically ill patients with Covid-19, an initial strategy of therapeutic-dose anticoagulation with heparin increased the probability of survival to hospital discharge with reduced use of cardiovascular or respiratory organ support as compared with usual-care thromboprophylaxis [1]. These three clinical trials 
also reported that among critically ill patients with COVID-19, an initial strategy of therapeuticdose anticoagulation with unfractionated or low-molecular-weight heparin was not associated with a greater probability of survival to hospital discharge or a greater number of days free of cardiovascular or respiratory organ support than was usual-care pharmacologic thromboprophylaxis [2]. The study observed the same findings of patients with COVID-19 who were already on anticoagulation, even before the diagnosis of COVID-19, and published that those patients had almost near zero mortality rates [3]. This research was submitted on August 15th, 2020, in the Journal of Mississippi State Medical Association [3]. These three clinical trials finding validate the author's observation why patients already on anticoagulation with Apixaban or Rivaroxaban were surviving as the patients were non critically ill or even asymptomatic when they were taking anticoagulation orally.

According to the Johns Hopkins coronavirus resource center, case fatality rates for COVOD-19 is $1.7 \%$ in the United States as of August $17^{\text {th }}, 2021$ [4]. It is standard of care to treat any hospitalized patient with prophylaxis dose of Enoxaparin (40 mg SubQ daily) or Heparin (5000-unit subQ TID) unless there is a contraindication. It is possible that this low dose of anticoagulation did help reduce case fatality rates in the US have one of the lowest mortality rates for COVID-19. Low molecular weight anticoagulants are weight-based drugs, and it is also possible that those had high fatality due to COVID1-9 were obese and the low dose anticoagulation was inadequate to provide any survival benefit. The goal of this pilot study was to develop a mechanism of death among patient with COVID-19 and subsequently develop a COVID-19 treatment algorithm based on risk of bleeding due to anticoagulants. 


\section{Method:}

The method of the study has been published elsewhere [5]. This study retrospectively evaluated the COVID-19 admission at Jackson Hospital, Alabama, USA from June 15th, 2020, to June 15th, 2021. The author's previous publication did not assess mechanism of death in patient with COVID-19 $[3,5]$. In addition, the other goal of the retrospective pilot study was to evaluate the risk of bleeding due to anticoagulation and dosing anticoagulation based on Kabir Bleeding Risk Calculator.

\section{Results:}

This study evaluated a variable dose of anticoagulation based on trends of $d$-dimer and found that intermediate or high dose enoxaparin is associated with better patient outcome and frequently patients do not need therapeutic anticoagulation for survival from COVID-19 [5]. The best treatment strategy from the retrospective study was starting hospital COVID-19 floor patients on Enoxaparin $0.5 \mathrm{mg} / \mathrm{kg}$ subq twice a day (for non-critical patients) and titrating dose up if the patient's condition deteriorates or if d-dimer worsened. The prior study did show that majority of the patient did not require full dose anticoagulation and an intermediate or low dose anticoagulation provided adequate survival benefits.

Based on the retrospective study results, the author developed COVID-19 mechanism of death

(Figure 1). Prior to death, patient with COVID-19 develops severe hypoxia and hypotension (step 6) which may look like septic shock, but it may be due to widespread alveolar capillary 
microthrombi (step 5). The microthrombi load in the body is reflected by increasing trends of Ddimer (step 5) which is been reported in in the prior study [4]. This increasing trends of D-dimer and hypoxia is also responsive to increased dose of anticoagulation indicating that D-dimer is related to massive micro thrombi in the pulmonary microcapillary. Early initiation of anticoagulation prevents worsening of hypoxia indicates widespread microthrombi formation not happening which indicates step 3 as an intermediate step among COVID-19 patients. A previous study already demonstrated that the COVID-19 virus binds with ACE 2 receptor in the vascular system which is step 1 in the mechanism [6]. It is a logical next step for COVID-19 to cause endotheliosis while entering endothelial cell by causing endothelial cellular injury (step 2) which leads to widespread coagulation cascade (step 3). These six steps show the mechanism of death among patient with COVID-19.

Figure 1: Kabir Hypothesis of COVID-19 Mechanism of Death. 


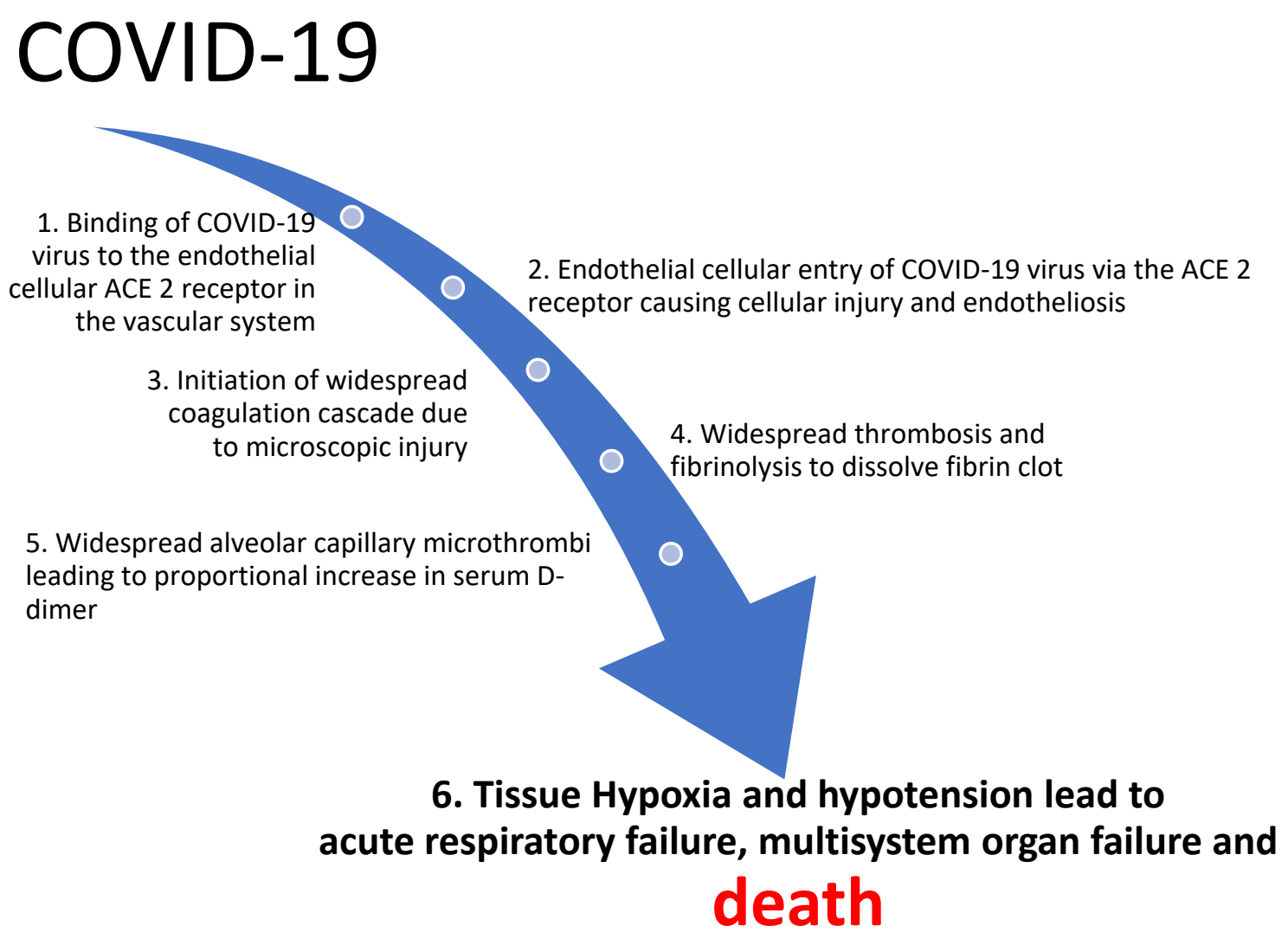

\section{Discussion:}

Widespread alveolar capillary microthrombi is the primary cause of COVID-19 death. Those patients who were admitted at the hospital medical floor (non-critically ill) have significant mortality benefit with early initiation of high dose anticoagulation. Those patients who were treated with higher dose of anticoagulation in the early stage of COVID-19 and later moved from the hospital floor to the intensive care unit have a higher chance of recovery compared to those who did not receive any anticoagulation prior to their critical care unit admission. The reason behind those failures is that anticoagulation does not dissolve microthrombi (blood clots) rather prevents their further growth. Treating patients with anticoagulation only can benefit when capillaries are still open (not clogged due to microthrombi). Those patients present in hospitals 
needing nasal cannula oxygen or high flow nasal cannula oxygen and or patients with low or moderately high D-Dimer (less than two-fold higher than the upper limit of normal) usually survive with anticoagulation and steroid combination treatments. The study observed that increasing trends of D-Dimer in any patient were inversely proportional to the survival rates and or clinical recovery from COVID-19 and vice versa. There is a dose-effect relationship between the decreasing trends of D-Dimer with the used anticoagulation dose.

These COVID-19 hospital floor patients provided unique experiences because of their early stages of disease presentation compared to patients admitted in the intensive care units. The study observed COVID-19 patients who presented in the late stages of COVID-19, or those who were directly admitted to the intensive care unit due to intubation or BiPAP requirements, were more likely to have poor outcomes compared to patients presenting at an earlier stage in the COVID hospital floor. Due to poor outcomes with therapeutic anticoagulation in the intensive care unit patients, nationwide intensivists were reluctant to adapt therapeutic anticoagulation in treating COVID-19. The dilemma is mostly rooted in anticoagulation related risk of bleeding and poor patient outcome. This biased experience of intensive care unit physicians leads to higher deaths among COVID-19 patients since the beginning of the pandemic.

If a state or country's healthcare system is reaching a breaking point it will be too late for getting vaccination to reduce COVID-19 cases as it takes more than four weeks for the vaccination to start working. In addition, there are increasing cases of break through cases of COVID-19 happening though vaccinated are 40 times more like to survive COVID-19 compared to unvaccinated. A contingency plan should be to start a nation or state awareness campaign asking 
patients to come to seek healthcare at urgent care or primary care clinics so that the chance of capturing early noncritical COVID-19 cases is very high. Again, if patients present to the emergency room in a critical stage, there will not be any benefit of therapeutic anticoagulation. Given the clinical trial [1] findings among noncritical COVID-19 treated with anticoagulation showed reduced use of cardiovascular or respiratory organ support, treating patient at diagnosis with oral therapeutic anticoagulation like Apixaban or Rivaroxaban may reduce need for hospitalization among patient with COVID-19.

Given, all anticoagulant reduces thrombotic risk and at the same time increase the risk of bleeding, the decision to administer an anticoagulant should be based on the assessment of the bleeding risk for the specific patient at a specific point in time. According to the current study, not all patients need therapeutic anticoagulation to treat COVID-19, a lower dose may be sufficient for those with low D-Dimer level. The authors previous study recommends the ideal way to dose anticoagulation is to titrate anticoagulation based on D-Dimer trends [5].

The dilemma of using therapeutic anticoagulation versus the treatment of COVID-19 without anticoagulation to prevent bleeding related mortality and morbidity can be solved if anticoagulation is used judiciously. The author developed the Kabir bleeding risk Score based COVID-19 treatment protocol showed in Table 1. The Kabir bleeding risk calculator can also be visited by clicking the following website: <https://patient.ddxrx.com/anticoagulation.php>. The author utilized available literature to develop the Kabir bleeding risk calculator [7-9]. 
Table 1: Developing Kabir risk of bleeding score with anticoagulation based on known risk factors: Treating noncritically ill COVID-19 patients.

\begin{tabular}{|l|l|}
\hline RISK FACTORS FOR BLEEDING WITH ANTICOAGULATION & LIKELIHOOD \\
\hline MALE SEX & 0.4 \\
\hline OLDER AGE ( $\geq 80 ; 40$ to 80; 18 to 40) & $0.75 ; 0.5 ; 0 ;$ \\
\hline RACE (ASIAN, HISPANIC, AFRICAN AMERICAN, WHITE) & $1 ; 0.5 ; 0.5 ; 0 ;$ \\
\hline CURRENTLY TREATED FOR PEPTIC ULCER DISEASE & 1 \\
\hline BLEEDING EPISODE WITHIN THE PAST 3 MONTHS & 1 \\
\hline PLATELET COUNT <50,000 & 1 \\
\hline ADMISSION IN ICU OR CCU & 0.6 \\
\hline EGFR <30; 30 TO 59; 60 & $0.5 ; 0.4 ; 0 ;$ \\
\hline INR >1.5 & 0.5 \\
\hline ACTIVE CANCER & 0.5 \\
\hline HISTORY OF RHEUMATIC DISEASE & 0.25 \\
\hline PRESENCE OF CENTRAL VENOUS CATHETER & \\
\hline PRIOR HISTORY OF STROKE & 0.5 \\
\hline
\end{tabular}




\begin{tabular}{|l|l|}
\hline PRIOR HISTORY OF GASTROINTESTINAL BLEEDING & 0.25 \\
\hline RECENT MYOCARDIAL INFARCTION & 0.25 \\
\hline DIABETES MELLITUS TYPE 2 & 0.25 \\
\hline
\end{tabular}

If the calculated bleeding score is zero based on Kabir risk score calculator in any patients with COVID-19 or COVID-19 like symptoms, the author suggests starting high dose anticoagulation while titrating anticoagulation dose up or down based on D-Dimer trends (Table 2).

Table 2: COVID-19 treatment strategy based on Kabir bleeding risk scores.

\section{RISK STRATIFICATION RECOMMENDATION}

LOW RISK (SCORE 0): Consider starting high-dose anticoagulation for COVID-19 upon diagnosis. For outpatients, consider therapeutic dose direct acting oral anticoagulant (i.e., Apixaban or Rivaroxaban etc.); For hospitalized patients consider Enoxaparin $0.5 \mathrm{mg} / \mathrm{kg}$ or $1 \mathrm{mg} / \mathrm{kg}$ dosing based on the initial level of D-Dimer and adjust dose based on D-Dimer trends.

INTERMEDIATE RISK Consider starting intermediate-dose or high-dose anticoagulation for (BLEEDING SCORE IN BETWEEN 0 - 2): COVID-19 upon diagnosis. For outpatients, consider Apixaban 5mg PO BID (adjust based on age or eGFR). For hospitalized patients, consider Enoxaparin $0.5 \mathrm{mg} / \mathrm{kg}$ subQ BID and adjust the dose up or down based on D-Dimer trends. 
HIGH RISK (BLEEDING SCORE > 2):
Consider starting low dose or intermediate-dose anticoagulation for COVID-19 upon diagnosis. For outpatients, consider low dose Apixaban $2.5 \mathrm{mg}$ PO BID. For hospitalized patients, consider Enoxaparin low dose or intermediate dose $(40 \mathrm{mg}$ subQ daily or 0.5 $\mathrm{mg} / \mathrm{kg}$ subQ BID) and adjust the dose up or down based on D-Dimer trends.

It is very important to discuss the risk benefit scenario with patient and family members prior to starting therapeutic anticoagulation among patients with intermediate to high-risk bleeding scores. Starting intermediate dose enoxaparin for select patients with high-risk scores is a better strategy than blanket therapeutic anticoagulation in terms of reducing mortality and morbidity related to bleeding associated with anticoagulation as the study showed majority of the patients did not require therapeutic dose anticoagulation for increased survival benefit.

\section{Conclusion:}

The author developed the Kabir bleeding risk score-based treatment strategies for COVID-19 patients which can be visited by clicking on the following link: <https://patient.ddxrx.com/anticoagulation.php>. Mortality and morbidity related to COVID-19 can be further reduced by starting anticoagulation based on Kabir bleeding risk score-based treatments. A reduced dose of anticoagulation is suggested if Kabir bleeding risk score shows intermediate or high-risk of bleeding. In such cases, anticoagulation dose can be titrated up or down based on trends of D-Dimers which will give an opportunity to further reduce bleeding risk among patients who are treated with anticoagulation. Further study is recommended to evaluate 
whether creating a statewide awareness about getting treatment with anticoagulation as soon as COVID-19 symptoms appears will leads to further decrease in mortality.

Acknowledgement: Abul Hussam, Ph.D. inspired me to conduct the research.

Ethical approval: The research related to human use has been complied with all the relevant national regulations, institutional policies, and in accordance the tenets of the Helsinki Declaration and has been approved by the authors' institutional review board of Jackson Hospital, Montgomery, Alabama.

Informed consent: All patients were admitted to the hospital as they meet hospitalization criteria and gave signed consent to treatments with any FDA approved drugs.

Conflict of Interest: The author has no conflict of interest to disclose.

\section{References:}

1. The ATTACC, ACTIV-4a, and REMAP-CAP Investigators. (2021) Therapeutic Anticoagulation with Heparin in Noncritically III Patients with Covid-19. $N$ Engl J Med DOI: 10.1056/NEJMoa2105911.

2. The REMAP-CAP, ACTIV-4a, and ATTACC. (2021) Therapeutic Anticoagulation with Heparin in Critically III Patients with Covid-19. N Engl J Med DOI: 10.1056/NEJMoa2103417.

3. Kabir, Azad. Letter to the editor. (2020): Is Therapeutic Anticoagulation the Answer in Treating COVID-19? JMSMA. October 2020; Vol 61 (10). 1-2 (Submitted August 15, 2020).

4. Johns Hopkins Coronavirus Resource Center: https://coronavirus.jhu.edu/data/mortality 
5. Kabir, Azad A. "Anticoagulation is the answer in treating noncritical COVID-19 patients" Open Medicine, vol. 16, no. 1, 2021, pp. 1486-1492. https://doi.org/10.1515/med-2021-0354

6. Li W, Moore MJ, Vasilieva N, Sui J, Wong SK, Berne MA, Somasundaran M, Sullivan JL, Luzuriaga K, Greenough TC, Choe H, Farzan M. Angiotensin-converting enzyme 2 is a functional receptor for the SARS coronavirus. Nature. 2003 Nov 27;426(6965):450-4. doi: 10.1038/nature02145. PMID: 14647384; PMCID: PMC7095016.

7. Decousus $\mathrm{H}$, Tapson VF, Bergmann JF, et al. Factors at admission associated with bleeding risk in medical patients: findings from the IMPROVE investigators. Chest 2011; 139:69.

8. Schünemann HJ, Cushman M, Burnett AE, et al. American Society of Hematology 2018 guidelines for management of venous thromboembolism: prophylaxis for hospitalized and nonhospitalized medical patients. Blood Adv 2018; 2:3198.

9. Adapted from Beyth RJ, Quinn LM, Landefeld CS. Prospective evaluation of an index for predicting the risk of major bleeding in outpatients treated with warfarin. Am J Med 1998; 105:91. 